\title{
Insect and Mammalian Innate Immune Responses Are Much Alike
}

\author{
Studying insect innate immunity sidesteps complexities of adaptive \\ immunity and could help in identifying antimicrobial drugs
}

\author{
Kevin Kavanagh and Emer P. Reeves
}

I nsects are found in almost every habitat on Earth, with as many as $10^{18}$ live individuals belonging to perhaps 1 million separate species. In terms of evolution, although insects and vertebrates diverged approximately 500 million years ago, many components of their biochemistries and physiologies remain similar. Moreover, because the innate immune systems of mammals and insects have several features in common, studying the insect immune system can yield insights into the functioning of the mammalian system without requiring sifting through the complexities of adaptive immunity in the latter.

In particular, both insect hemocytes and mammalian neutrophils function similarly, engulfing and killing pathogens. Moreover, both types of cell produce superoxide, using extraordinarily similar p47 and p67 proteins that, in both cases, are translocated from the cytosol to the plasma membrane where they help to form a key oxidase. Based on such similarities, insects can be used for screening pathogens as well as candidate drugs that help to control them, thereby reducing the number of mammals that are used for these purposes.

Senior Lecturer in

the Department of

Biology, National

University of Ire-

land, Maynooth,

County Kildare, Ire-

land, and Emer $P$.

Reeves is Senior

Lecturer in the Department of Medicine, Royal College of Surgeons in Ireland, Beaumont Hospital, Dublin, Ireland.

\section{Studying Insect Innate Immune Response}

We use larvae of the greater wax moth Galleria mellonella to determine the relative virulence of Candida albicans isolates and to differentiate between this and other pathogenic or nonpathogenic yeast species. Further, the virulence of C. albicans mutants tested in larvae of G. mellonella correlates with mutant virulence in BalbC mice. Similarly, toxin production correlates with virulence of the pulmonary pathogen $A s$ pergillus fumigatus for larvae of G. mellonella (Fig. 1). In addition, the stages of conidial germination are directly associated with virulence of $A$. fumigatus in this insect. We use several endpoints to measure the effects of various pathogens on G. mellonella. They include fluctuations in fungal load and haemocyte density, as well as differential expression of genes for specific antimicrobial peptides.

In addition to the larvae of G. mellonella, other insects, including silkworms, the fruit fly Drosophila melanogaster, and the nematode Caenorhabditis elegans (see p. 600), are being used to assess the virulence of a range of pathogens, including Pseudomonas aeruginosa, and for determining the potency of antimicrobial agents. Insects are also being employed to measure the virulence of clinical isolates of several bacterial pathogens, including $P$. aeruginosa, Proteus mirabilis, Escherichia coli, Bacillus cereus, and Staphylococcus aureus.

Insects are being used to assess virulence that is altered through deleting or over-expressing genes within a range of pathogens. Moreover, the efficacy of antibiotics can be tested by deter-

\section{Summary}

- The innate immune systems of mammals and insects have several features in common.
- Both insect hemocytes and mammalian neutrophils engulf and kill pathogens, and both types of cell produce superoxide, using extraordinarily similar p47 and p67 proteins.

- Larvae of the greater wax moth Galleria mellonella provide a model system for evaluating familiar bacterial and fungal pathogens as well as for testing candidate antimicrobials.

- Insect immune systems function cooperatively and exhibit both humoral and cellular components. 
mining how well they block or kill bacteria that can infect silkworm larvae. Such results correlate with antibiotic efficacy measurements in mice.

\section{Advantages in Using Insects Such as G. mellonella To Assess Virulence}

Assessing microbial virulence in insects offers several advantages over doing so in vertebrates. For instance, insects are inexpensive to raise and to house compared to rodents or other mammals. Moreover, although the innate immune system of insects resembles that of mammals, insects lack an adaptive immune system, thus avoiding its complications in following immune responses to pathogens.

Commercially available larvae of $G$. mellonella are easy to inoculate via injection into the haemocoel through the last left pro-leg. The base of the pro-leg can be opened by applying gentle pressure to the sides of the leg, and it reseals without leaving scars. Larvae can be stored at $15^{\circ} \mathrm{C}$ prior to use and, once inoculated, may be maintained at temperatures up to $37^{\circ} \mathrm{C}$ along with appropriate controls to track survival. Larvae are handled carefully to ensure survival and avoid expression of stress proteins. In using G. mellonella larvae assays, we monitor endpoints, including survival following inoculation, fluctuations in haemocyte density, and changes in expression of genes encoding antimicrobial proteins following infection. Although tracking larval death is easy and straightforward, following other more labor-intensive endpoints provides valuable insights into host immune responses.

\section{Insect Immune Response}

Insect immune systems function cooperatively, and have both humoral and cellular components. The humoral component produces antimicrobial peptides (AMP). Although primitive, AMPs are both competent and widespreadfound, for example, among vertebrates, insects, and plants. AMPs are hydrophobic, typically less than $10 \mathrm{kDa}$, and active on membranes of pathogenic microorganisms, recognizing acidic phospholipids on the surface of bacterial or other membranes.

G. mellonella produces several distinct types of AMP, including an inducible metalloproteinase inhibitor (IPMI), transferrin, galiomicin, and gallerimycin, all of which play important roles in its humoral response. Without signal-depen- dent synthesis of AMPs, insects would succumb quickly to microbial infections. When G. mellonella larvae are inoculated with a sublethal dose of C. albicans or with fungal cell wall components, AMP gene expression increases and the larvae produce amounts of antimicrobial peptides that render them capable of dealing with potentially lethal doses of this pathogenic yeast.

The cellular immune response of insects consists of rapid synthesis and moblilization of immune cells, called hemocytes, that engulf invading pathogens. The six known types of hemocytes include plasmatocytes, granulocytes, prohaemocytes, coagulocytes, spheruloctes, and oenocytoids. Hemocytes bear a number of structural and functional similarities to neutrophils, which play a critical role in the mammalian innate immune response to microbial pathogens (Fig. 2).

\section{Insect Hemocytes Play a Central Role in Immune Response}

Like the neutrophils of mammals or other vertebrates, insect hemocytes play a central role in their innate immune response to infectious agents, engulfing, internalizing, and destroying invading microorganisms. When either mammalian neutrophils or insect hemocytes are activated, there is a burst in reactive oxygen species (ROS) that is accompanied by reduction of cytochrome $c$. Specifically, hemocytes of G. mellonella consume oxygen and produce superoxide $\left(\mathrm{O}_{2}{ }^{-}\right)$at the rate of $0.25 \mu \mathrm{M} / \mathrm{min} / 10^{6} \mathrm{In}$ hemocytes, the $\mathrm{O}_{2}{ }^{-}$-generating NADPH oxidase complex consists of a membrane-bound flavocy- 


\section{FI G U E 2}

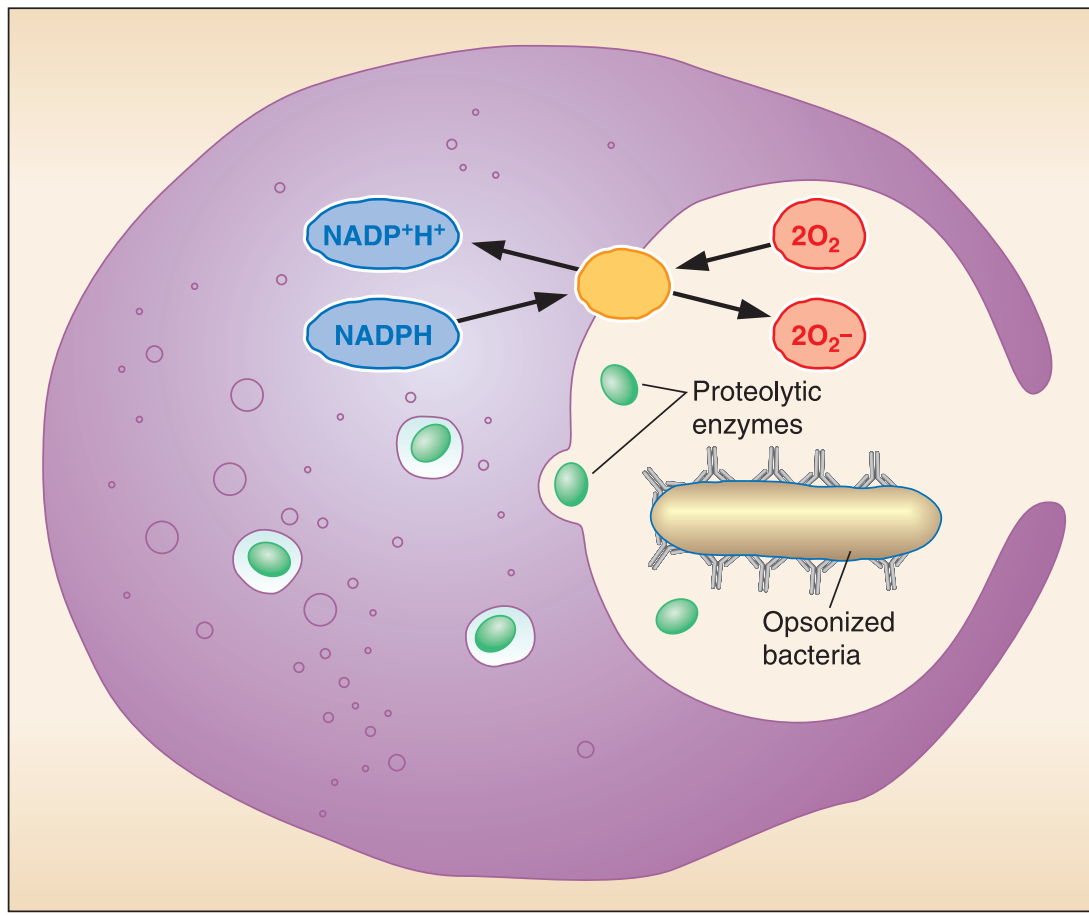

Neutrophil-mediated killing of engulfed microbe. Microbes are killed by the production of superoxide and the release of proteolytic enzymes into the vacuole.

Among individuals with chronic granulomatous disease (CGD), oxidase is missing or has very low activity. Thus, such individuals fail to produce $\mathrm{O}_{2}{ }^{-}$when infected with a range of microbial pathogens, including $S$. aureus, Klebsiella, E. coli, Pseudomonas, Serratia marcescens and also fungi, especially Aspergillus fumigatus.

\section{NADPH Oxidase of Neutrophils and Hemocytes}

We established that hemocytes of the greater wax moth, G. mellonella, can phagocytose bacterial and fungal cells. Moreover, the kinetics of phagocytosis and microbial killing in insect hemocytes and human neutrophils are similar. Diphenyleneiodonium chloride (DPI), which inhibits NADPH oxidase, blocks both superoxide production and microbial killing in both cell types.

The G. mellonella hemocytes contain several proteins that are homologous to human neutrophil phox proteins, including gp91 $91^{\text {phox }}, \mathrm{p} 67^{\text {phox }}, \mathrm{p} 47^{\text {phox }}$, and the GTP-binding protein rac 2 . Moreover, the 47- and 67-kDa insect

tochrome $b_{558}$ (composed of two proteins, $\mathrm{p} 22^{\text {phox }}$ and gp91 $\left.{ }^{\text {phox }}\right)$ and four cytosolic factors, $\mathrm{p} 47^{\text {phox }}, \mathrm{p} 67^{\text {phox }}, \mathrm{p} 40^{\text {phox }}$, and the small G protein, rac 2 (Fig. 3). These cytosolic proteins interact with one another, with rac, and with the flavocytochrome through a number of Src homology 3 (SH3) proteins containing prolinerich tetratricopeptide repeats and PC motifs.

This oxidase system is subject to stringent regulation at infectious and inflammatory sites. The cytochrome $b_{558}$ electron transport system forms the membrane-docking site for translocated cytosolic components of the oxidase system. Phosphorylation-induced conformational changes in $\mathrm{p} 47^{\mathrm{phox}}$ target interactions between the $\mathrm{SH} 3$ domain and the proline-rich region of $\mathrm{p} 22^{\text {phox }}$ in a step that is essential for attaching the translocated $\mathrm{p} 47^{\text {phox }}, \mathrm{p} 67^{\text {phox }}$, and $\mathrm{p} 40^{\text {phox }}$ components to the flavocytochrome complex. Meanwhile, rac 2 translocates to the membrane, where it also interacts with the flavocytochrome complex and with $\mathrm{p} 67^{\text {phox }}$. The activated cytochrome takes electrons from NADPH and passes them, via FAD and heme, to oxygen. proteins are distributed throughout the cytosol and in the perinuclear region in a pattern similar to that seen in neutrophils. Further, the hemocyte $67-$ and $47-\mathrm{kDa}$ proteins contain peptides that match those in the $\mathrm{p} 67^{\text {phox }}$ and $\mathrm{p} 47^{\text {phox }}$ of human neutrophils. For example, the p67 of insect haemocytes contains 10 peptides that are identical to corresponding peptides in $\mathrm{p} 67^{\text {phox }}$ of neutrophils, while insect p47 contains 7 peptides that are homologous to $\mathrm{p} 47^{\mathrm{phox}}$ of human neutrophils. Thus, despite insects and vertebrates diverging almost 500 million years ago, key features of these two proteins are conserved.

When $\mathrm{p} 47^{\text {phox }}$ and $\mathrm{p} 67^{\text {phox }}$ in mammalian neutrophils translocate from the cytosol to the membrane, they activate NADPH oxidase. We find that PMA stimulates the homologous hemocyte $67-$ and $47-\mathrm{kDa}$ proteins to translocate from the cytosol to the plasma membrane. When the inhibitor DPI is added to haemocytes to block oxidase activity, translocation is also halted. Both DPI and another inhibitor, gliotoxin, block translocation of $\mathrm{p} 67^{\mathrm{phox}}$ and p $47^{\text {phox }}$ in human neutrophils. 


\section{F I G U R E 3}

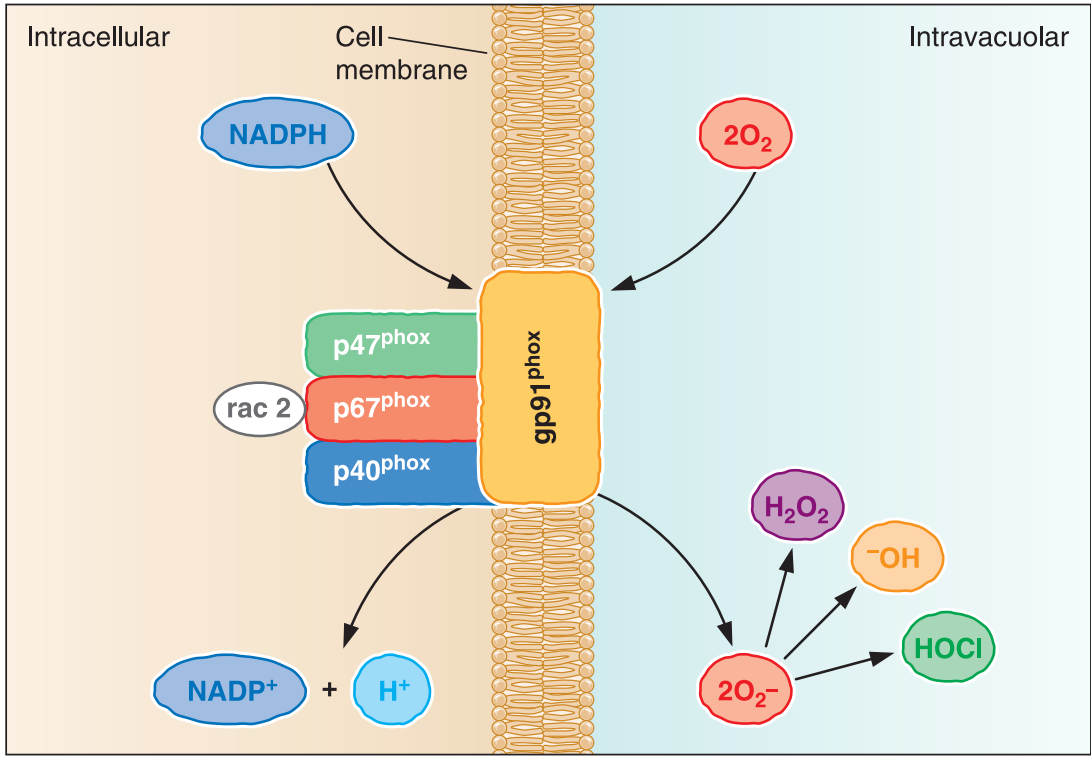

Superoxide production in neutrophils. Superoxide production is mediated by the NADPH oxidase complex, which consists of a number of constituent proteins.

\section{ACKNOWLEDGMENTS}

Our work has been supported by funding from the Higher Education Authority of Ireland under the Programme for Research in Third Level Institutes 3 (2002-2007).

\section{SUGGESTED READING}

Bergin, D., E. P. Reeves, J. Renwick, F. B. Wientjes, and K. Kavanagh. 2005. Superoxide production in haemocytes of Galleria mellonella-identification of proteins homologous to the NADPH oxidase complex of human neutrophils. Infect. Immun. 73: 4161-4173.

Bergin, D., L. Murphy, J. Keenan, M. Clynes, and K. Kavanagh. 2006. Pre-exposure to yeast protects larvae of Galleria mellonella from a subsequent lethal infection by Candida albicans and is mediated by the increased expression of antimicrobial peptides. Microbes Infect. 8:2105-2112.

Brennan, M., D. Y. Thomas, M. Whiteway, and K. Kavanagh. 2002. Correlation between virulence of Candida albicans mutants in mice and Galleria mellonella larvae. FEMS Immunol. Med. Microbiol. 34:153-157.

Fuchs, B. B., and E. Mylonakis. 2006. Using nonmammalian hosts to study fungal virulence and host defence. Curr. Opin. Microbiol. 9:346-351.

Hamamoto, H., K. Kurokawa, C. Kaito, K. Kamura, I. Manitra Razanajatovo, H. Kusuhara, T. Santa, and K. Sekimizu. 2004. Quantitative evaluation of the therapeutic effects of antibiotics using silkworms infected with human pathogenic microorganisms. Antimicrob. Agents Chemother. 48:774-779.

Kavanagh, K and E. P. Reeves. 2004. Exploiting the potential of insects for the in vivo pathogenicity testing of microbial pathogens. FEMS Microbiol. Rev. 28:101-112.

Reeves, E., H. Lu, J. Hugues Lortat, C. Messina, S. Bolsover, G. Gabella, E. Potma, W. Warley, J. Roes, and A. Segal. 2002. Killing activity of neutrophils is mediated through activation of proteases by K+ flux. Nature 416:291-296.

Renwick, J., E. P. Reeves, F. B. Wientjes, and K. Kavanagh. 2007. Translocation of proteins homologous to human neutrophil p47phox and p67phox to the cell membrane in activated hemocytes of Galleria mellonella. Dev. Comparative Immunol. 31:347-359.

Salzet, M. 2001. Vertebrate innate immunity resembles a mosaic of invertebrate immune responses. Trends Immunol. 22:285-288. Scully, L. R., and M. Bidochka. 2006. Developing insect models for the study of current and emerging human pathogens. FEMS Microbiol. Lett. 263:1-9. 